\title{
Potential of deconcentration of attention methods for use in the information society
}

\author{
Alexey Ermakov ${ }^{1,2^{*}}$ \\ ${ }^{1}$ Russian State University of Physical Education, Sport, Youth and Tourism (SCOLIPE), Moscow, \\ Russia \\ ${ }^{2}$ Federal Scientific Center of Physical Culture and Sports, Moscow, Russia
}

\begin{abstract}
This paper is devoted to the results of our issues exploration increasing efficiency action in extreme circumstances. Long time we study only combat oriented activities but now it is obvious that work with information torrents has the same features of stress as a fight. Our exploration was based on Oleg Bakhtiyarov ideas who study deconcentration of attention phenomenon. We adapted these ideas and combined traditional for our country approaches to hand-to-hand combat and special software. We used two main ideas for experiments. The first one was searching for hidden threats and stealthy attacks with deconcentration and after that was concentration attention on threats and destroying them. This gave us only part-time at deconcentration. We used "plane deconcentration" for searching and "multidimensional deconcentration" for action. The second idea was about using deconcentration for avoiding dangerous situations. That case was about full-time deconcentration. We discovered two types of usage deconcentration indirect or without direct contact with attackers but not only this feature and we need to explore it in the future. We discovered the same features in combat activities and works with information torrents. In addition, the same features were discovered between our data and the other from e-sports. In addition, the same features were discovered between our data and the other from e-sports. All of that gives us opportunities to use the deconcentration of attention methods for tasks that are needed for operators and common people in the information society (searching, detection, distinguishing, and filtration of information torrent) and, of course, for mental hygiene goals.
\end{abstract}

\section{Introduction}

Long time we are experimenting on various groups of people using deconcentration of attention methods [5-8]. These were military members, law enforcement officers, athletes, and students with the majority was sports training. The explorer who gets a lot of experience in low-intensity conflicts that were so often after the USSR demolition inspired the beginning of this activity. It was psychologist Oleg Bakhtiyarov [1, 3]. He used methods training of Soviet Union operators in space programs for consulting the members

*Corresponding author: bigbr@mail.ru 
of low-intensity conflicts [2]. During work with the regular and irregular participants of combat, he discovered differences in the organization of attention these categories of the combatants. Irregular participants of combat without special military training very often demonstrated phenomena deconcentration of attention. It was definitely peculiar these phenomena were the more explicitly the more successful combat activity of test subject. At that time in our university was functioning department theory and methodology applied training and extreme activities, and assuredly we were been interested in comprehensively studying the applied effects these phenomena [5-8].

Natalia Molchanova conducted one of the explorations for safety freedivers, scuba divers, and other waters activities. This exploration was based on ideas deconcentration of attention methods could reduce the level of extreme nervousness, fear or dread. It turned out to be useful for applying in various situations. For example, the elaborated methods were successfully used to psychological preparation students before exams and tests. It can even show us possibilities usage such methods for enhanced effectiveness functioning in the information society when information torrents could provoke negative emotions and ineffectiveness.

Another way of attention methods apply deconcentration was used by our group for exploring approaches to the increase effectiveness operators in the close quarters combat (CQC), hand-to-hand combat, and self-defence situation. This paper is devoted our results of such ways to deconcentration of attention methods.

\section{Materials and methods}

We were firstly interested in the increasing effectiveness of operators' activity. For this reason, we had to organize a model of the situation according to the reality of the combat conditions. For this reason, we organized our research as a complex system of events. Firstly, in each detached experiment, we have a phase of especial training. Secondly, we assembled, adopted and elaborated various set tests according to explores goals. Today we discuss two of our main ideas of attention methods usage deconcentration.

The first of them was conversion deconcentration to concentration [5-7]. The operators firstly had to search for hidden threats and stealthy attacks and secondly was concentration attention on threats and destroying them. The second idea was about permanent activity in deconcentration of attention. Operators had to avoid danger and escape a dangerous zone $[8]$.

The first experiment had two stages. The first stage contains training in "plane deconcentration". During this stage, operators obtain skills that distinguish slight visual signals. It would be on the computer screen. Operators pay more attention background than the figure and simultaneously make the right conclusions and make the right decisions about changes in the figure. The test for this stage was an exercise "threat in the row" when to operator approach a row of six men and only two of them have hidden weapons. Each step men prepare a weapon for attacking. The weapons are made more and more visually. Men without weapons make similar movements. The operator has to point armed men as quickly as possible. Results were determined according to quantity steps that were made by a row of attackers.

The second stage contains training in "multidimensional deconcentration". This training makes the process deconcentration more deeply. Operators use not only visual but also additionally audial, tactile deconcentration, and pay attention to the total surrounding space. The test for this stage contains adopted exercise. It was elaborated for Soviet Union Special Forces by A.I. Dolmatov [4]. In this test, the operator defends against five attackers. In the traditional test, there is an order of attacks, but in our case, all attacks were various and spontaneous. It is shown in Fig. 1. Results were determined according to the number of 
successful action operators against attackers. There are evaluated three types of mistakes. The first type is the mistakes of the wrong recognition of attackers. The second type is the mistakes of the wrong choice of the defending way. The third type is the pause between defence and counterattack. Only without such mistakes, we may note successful operators' action. In each stage, these tests were used before and after whole training. Except these tests were used special software for detecting level of deconcentration.

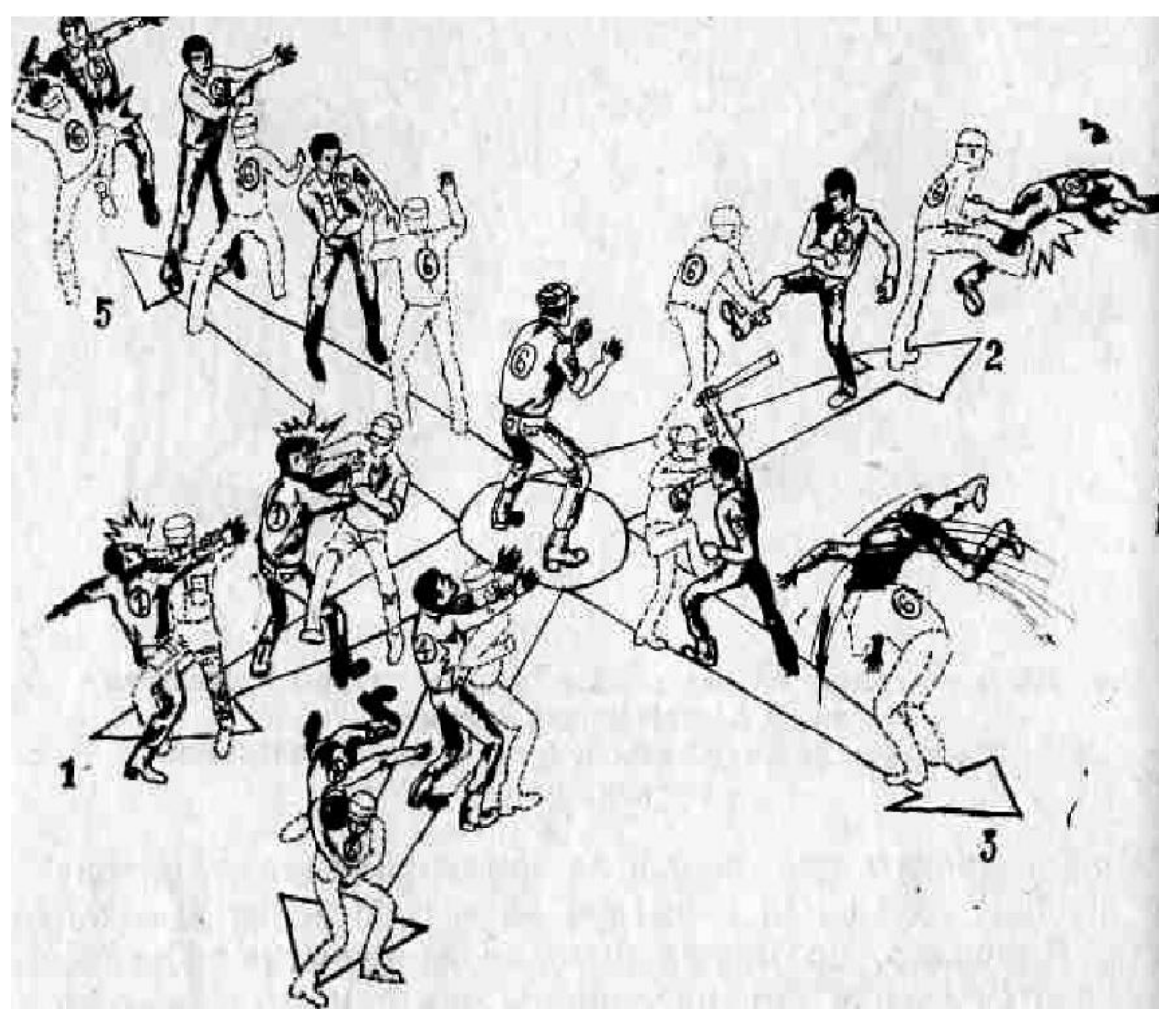

Fig. 1. The view of traditional test by A.I. Dolmatov (1987) [4].

Before we used only computer simulator "Romashka 1" ("daisy wheel") that is shown in Fig.2.a. In the computer simulator "Romashka 1» ("daisy wheel"), operator have to distinguish a circle that has another color. In this figure, the circle is very noticeable, but operators have to distinguish a circle that is not so simple discernible and color difference tiny that is shown in Fig.2.b.

Today we use another computer simulator "Shariki" ("Balls") that designed by V. Golubev. This simulator has a lot of tests and games. 


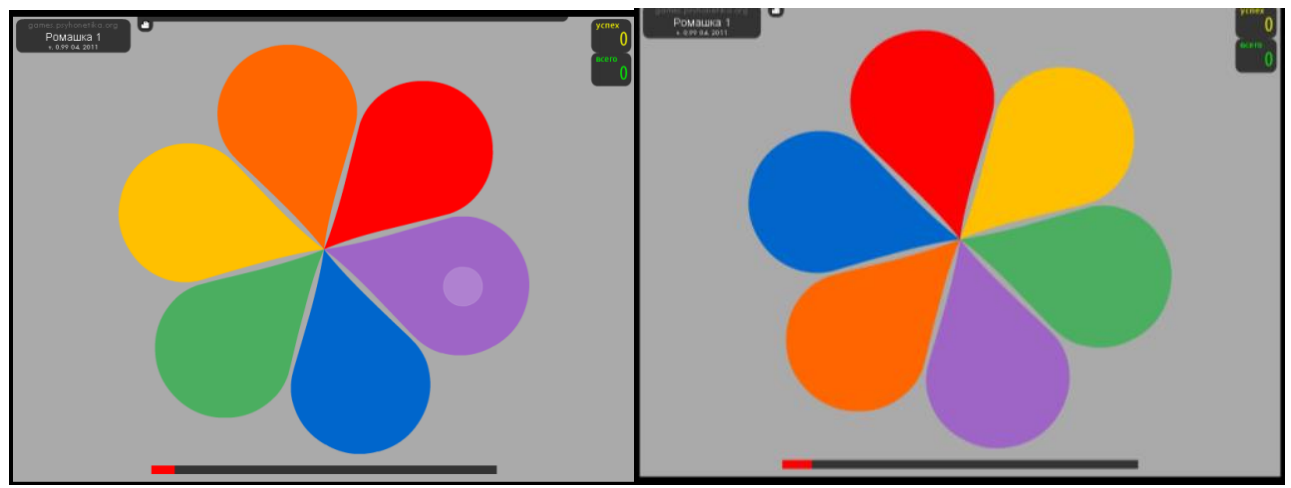

Fig. 2. The computer simulator "Romashka 1" ("daisy wheel") a. - simple mod; b.-hard mod

The second experiment had also two stages and the first of them also was devoted training of plane deconcentration but it took not so much time and it was only overview. After that training in "multidimensional deconcentration" as previously.

The new part was not about using deconcentration for detected objects future concentrations but for effective continuous activity with deconcentration. These were tests for avoiding dangerous situations. These tests had one task to avoid knife attacks. In the first of them, there were five attackers encircled the operator and only two of them had simulator knives. In the second there were ten attackers encircled, the operator and five of them had simulator knives. In both tasks, the operator was not aware of who is armed.

Besides that during all events, members were interviewed.

\section{Results and discussion}

Before these experiments, we explored Simple and complex sensomotor reaction for increasing efficiency operators in extreme but not only swiftness influences efficiency [9]. Now we obtain another way for increasing efficiency by using deconcentration of attention. For statistical analysis results, we used to Q-nonparametric statistical test by Rosenbaum. The outcome of our two series of experiments is shown in Table 1.

Table 1. Results differentiation two series of experiments according various tests.

\begin{tabular}{|c|c|c|c|}
\hline Name of test & $\begin{array}{c}\text { "Threat in the row" } \\
\text { (number of steps) }\end{array}$ & $\begin{array}{c}\text { "Romashka 1" } \\
\text { (number of } \\
\text { detections) }\end{array}$ & $\begin{array}{c}\text { Advanced test byA.I. } \\
\text { Dolmatov (number of } \\
\text { successful actions) }\end{array}$ \\
\hline $\begin{array}{c}\text { Validity of differences } \\
\text { the first series }\end{array}$ & $\begin{array}{c}\mathrm{Q} \text { empiric (22)> Q } \\
\text { critical (9) } \\
\mathrm{p} \leq 0.01\end{array}$ & $\begin{array}{c}\mathrm{Q} \text { empiric(22) }>\mathrm{Q} \\
\text { critical (9) } \\
\mathrm{p} \leq 0.01\end{array}$ & $\begin{array}{c}\mathrm{Q} \text { empiric }(8)>\mathrm{Q} \\
\text { critical (6) }\end{array}$ \\
\hline Name of test & $\begin{array}{c}\text { "Avoiding knives } \\
\text { attack 5" (number of } \\
\text { fail actions) }\end{array}$ & $\begin{array}{c}\text { "Avoiding knives } \\
\text { attack 10" (number } \\
\text { of fail actions) }\end{array}$ & $\begin{array}{c}\text { Advanced test byA.I. } \\
\text { Dolmatov (number of } \\
\text { successful actions) }\end{array}$ \\
\hline $\begin{array}{c}\text { Validity of differences } \\
\text { the second series }\end{array}$ & $\begin{array}{c}\text { Q empiric (8) }>\mathrm{Q} \\
\text { critical (6) } \\
\mathrm{p} \leq 0.05\end{array}$ & $\begin{array}{c}\text { Q empiric(24) }>\mathrm{Q} \\
\text { critical (9) } \\
\mathrm{p} \leq 0.01\end{array}$ & $\begin{array}{c}\text { Q empiric (8) }>\mathrm{Q} \\
\text { critical (6) }\end{array}$ \\
\hline
\end{tabular}

All tests were confirmed changes between control and experimental groups but the level was not equal. We can divide tests into two groups (with $\mathrm{p} \leq 0.01$ and $\mathrm{p} \leq 0.05$ accordingly). The main feature that divides groups is the type of contact with attackers (indirect or direct). This feature is connected with the type of deconcentration (plane or multidimensional). The first of group contains tests "Threat in the row" and "Romashka 1" what don't wonder because these tests use "plane deconcentration" but it is unexpected 
presence in this group the test "Avoiding knives attack 10" which demands "multidimensional deconcentration". We suppose so two circles of attackers decrease their level of organization and allow operator to obtain advantages. The second group contains tests: "Avoiding knives attack 10" and "Advanced test by A.I. Dolmatov".

Table 2. Results subjective assessment operators' separate features of activity.

\begin{tabular}{|c|c|c|}
\hline Name of features & $\begin{array}{c}\text { Without direct } \\
\text { contact with } \\
\text { attackers }\end{array}$ & $\begin{array}{c}\text { In direct contact with } \\
\text { attackers }\end{array}$ \\
\hline $\begin{array}{c}\text { Number objects of } \\
\text { control }\end{array}$ & $22 \%$ & $16 \%$ \\
\hline $\begin{array}{c}\text { The longtime } \\
\text { concentration of } \\
\text { attention }\end{array}$ & $22 \%$ & $10 \%$ \\
\hline $\begin{array}{c}\text { Complicating the } \\
\text { behaviour of objects }\end{array}$ & $20 \%$ & $13 \%$ \\
\hline $\begin{array}{c}\text { The velocity of change } \\
\text { situations }\end{array}$ & $16 \%$ & $28 \%$ \\
\hline $\begin{array}{c}\text { Negative } \\
\text { psychological effects }\end{array}$ & $11 \%$ & $8 \%$ \\
\hline Other features & $9 \%$ & $25 \%$ \\
\hline
\end{tabular}

This group strongly demands "multidimensional deconcentration" and it was more difficult to train. Interviews gave us information about subjective assessment operators' separate features of activity. It is shown in Table 2. In this data, we cannot observe problems with muscle, strength, stamina, et cetera. All problems are connected to information and only direct contact gave us physical features (velocity) which also can belong information.

According to this data, we can see similarities with the challenges of information society. We also have interviewed e-athletes and they distinguish similar difficulties in the information society.

Today we have a bulk of information that come at the same time from one or two sources (as PC or smartphone). This problem could be resolved by "plane deconcentration" but when we use one complicate the situation, for example, VR-technology that case demand "multidimensional deconcentration" to increase efficiency.

\section{Conclusions}

The results of this research give us opportunities to say that combat stress and information stress in modern society have the same features and we can use our way psychological skills and physical training for adaptation to the stress of the information society. It concerns not only extreme situations and training operators but also all work with information torrent. Such work per se is significant stress in the information society and it gives us opportunities to use deconcentration of attention methods for various goals (it could be useful for the following tasks: searching, detection, distinguishing, and filtration of information torrent). It can be used for helping students, for training e-sports or employees who deal with information, and of course for mental hygiene.

\section{References}

1. O.G. Bakhtiyarov, Deconcentration, 127 (2004). 
2. O.G. Bakhtiyarov, Problems of Military Psychology, 171-181 (2003)

3. O.G. Bakhtiyarov, R.R. Muslimov, Psychonetics and development of new information technologies (University conscious states and virtual reality research laboratory, Moscow), Electronic information systems, 2, 25-32 (2017) https://elibrary.ru/item.asp?id=29680153

4. A.S. Kuznetsov, Z.M. Kuznetsova, Russian Journal of Physical Education and Sport, 14(4), 5-7 (2019). DOI: 10.14526/2070-4798-2019-14-4-5-7

5. A.V. Ermakov, V.A. Damditsurunov, V.S. Piyannikov, Theory and Practice of Physical Culture, 4, 57-58. (2016) URL:https://www.elibrary.ru/item.asp?id=27192334

6. A.V. Ermakov, V.A. Damditsurunov, V.S. Piyannikov, R.R. Muslimov, Theory and Practice of Physical Culture, 1, 17 (2017) URL: https://elibrary.ru/item.asp?id=29655448

7. A.V. Ermakov, N. N. Zharikov. Physical culture in the system of professional education: ideas, technologies and prospects, 3, 96-101 (2018) URL: https://www.elibrary.ru/item.asp?id=35084022

8. A.V. Ermakov, V.A. Damditsurunov, V.S. Piyannikov, Theory and Practice of Physical Culture, 1, 30-33 (2018) https://elibrary.ru/item.asp?id=35162376

9. A.V. Ermakov, Simple and Complex Sensomotor Reaction for Choice when Teaching Protection Against Armed Attacker, FICEHS 2019 (France, 2019) DOI: https://dx.doi.org/10.2991/aebmr.k.200114.185 